\title{
A New Linear Magnetic Gear with Adjustable Gear Ratios and its Application for Direct-Drive Wave Energy Extraction
}

\author{
Wenlong Lia ${ }^{\mathrm{a}}$ K. T. Chau ${ }^{\mathrm{a}, *}$, Christopher H. T. Lee ${ }^{\mathrm{a}, \mathrm{b}}$, T. W. Ching ${ }^{\mathrm{c}}$, Mu Chen ${ }^{\mathrm{a}}$, and J.Z. Jiang ${ }^{\mathrm{a}}$ \\ ${ }^{a}$ Department of Electrical and Electronic Engineering, The University of Hong Kong, Hong Kong, China \\ ${ }^{\mathrm{b}}$ Research Laboratory of Electronics, Massachusetts Institute of Technology, Cambridge, USA \\ ${ }^{\mathrm{c}}$ Faculty of Science and Technology, University of Macau, Macao, China
}

In this paper, a new linear magnetic gear with multiple gear ratios is proposed. Unlike the conventional linear magnetic gears, the proposed one purposely utilizes the aluminum-nickel-cobalt (AINiCo) permanent magnets (PMs) as the excitation source. Due to the relatively low coercivity, AlNiCo PMs can be magnetized or demagnetized by applying an appropriate DC current pulse into the magnetizing winding. Thus, the pole-pair numbers of PMs on two movers can be varied accordingly so that different gear ratios can be achieved. Firstly, the mathematical modeling of the hysteresis loop of AlNiCo PMs is established. Then, the proposed linear magnetic gear with adjustable gear ratios is presented. Instead of the traditional surface-mounted PM configuration, the proposed machine installs the PMs using the surface-inset and consequent-pole configuration. Hence, the adverse influence during the magnetizing or demagnetizing process can be minimized. Since the magnetizing windings are placed in the movers, no position control is required for the magnetizing or demagnetizing process. By applying finite element analysis, the electromagnetic performances of the proposed linear magnetic gear with adjustable gear ratios are evaluated. Finally, the proposed linear magnetic gear is applied for directdrive wave energy extraction. Owing to the capability of adjustable gear ratios, the wave energy converter can be operated in resonance with the waves at different sea states so that the maximized power can be captured.

Index Terms-Permanent magnet, linear magnetic gear, variable gear ratio, direct-drive, wave energy.

\section{INTRODUCTION}

Owing to the increasing concern on the energy utilization as well as environmental protection, as one of the most promising candidates to relieve these problems, the magnetic gear has become a hot research topic since the past decades. Compared with the mechanical gear, the magnetic gear makes use of the magnetic field interaction which has no physical contacts between gear teeth. Consequently, it exhibits various distinct features, namely less mechanical 
wear and tear, high efficiency, less maintenance and silent operation. Throughout the years, various magnetic gears have been proposed and designed, such as the magnetic worm gears [1], the external-meshed magnetic gears with parallel axes [2], the magnetic bevel gears [3], the coaxial magnetic gears [4-6], the planetary magnetic gears [7, 8], the linear magnetic gears $[9,10]$, the trans-rotary magnetic gears [11], the axial-flux magnetic gears [12, 13], as well as the transverse-flux magnetic gears [14]. Owing to the fact that the flux density of permanent magnets (PMs) is uncontrollable, the pole-pair numbers of magnetic fields produced by PMs are unchangeable so that the gear ratios are also fixed. The single gear ratio of magnetic gears is ill-suited for many industrial applications, including electric vehicle propulsion and renewable power generation.

In order to solve the problem, the magnetic gears with adjustable gear ratios are suggested: Shah et al. proposed a variable speed magnetic gear using rotational control rotor [15], while Husain et al. utilized a set of armature winding to replace the PMs on one of the rotors [16]. By adopting the pole changing technique, the gear ratio can then be varied. Since the slip rings are required for current conduction in the wound rotor, it degrades the reliability of the whole system. To remove the slip rings and to improve the situation, a continuously variable speed vernier magnetic gear is proposed [17]. In particular, the armature winding is placed in the stationary part so that the frequency of the AC supply can be adjusted. According to this arrangement, the speed ratio can be varied while the torque ratio is unchanged. Atallah et al. proposed a magnetic power split device to achieve a magnetic continuously variable transmission function [18]. The 3-phase winding, which was implemented in the outer side of the magnetic gear, was equipped within the stator. This magnetic gear consists of one stator with armature winding and three rotors, namely the control rotor, the input rotor and the output rotor. By feeding a variable frequency current into the armature winding, the speed of the control rotor can be adjusted so that the gear ratio of the input rotor and the output rotor can be adjusted accordingly. However, the three rotors result in a three-airgap topology, which increases the structure complexity and reduces the machine reliability. Recently, a machine called "memory machine", which utilizes the aluminum-nickel-cobalt (AlNiCo) material as the excitation source, has been proposed. Upon the employment of AlNiCo PMs, the flux density of the memory machine can be online magnetized or demagnetized. By solely using a temporary current pulse, the AlNiCo PMs can be easily magnetized, demagnetized and reversely magnetized. Therefore, the PM pole-pair number of the memory machine can be adjusted flexibly, and this technique is also known as pole-dropping and pole-reversing [19]. By borrowing this concept, a magnetic gear with multiple gear ratios was proposed [20]. The AlNiCo PMs can 
be purposely magnetized or demagnetized so that the pole-pair numbers of PMs on the two rotors of the magnetic gears can be changed. Hence, the gear ratio can be adjusted consequently.

The purpose of this paper is to combine the linear magnetic gear topology and the flux-mnemonic technique to form a new linear magnetic gear topology with multiple gear ratios. In particular, by incorporating the AlNiCo PMs and the magnetizing winding set into the linear magnetic gear, the linear magnetic gear with adjustable gear ratios can be accomplished.

\section{DESIGN}

The linear magnetic gear consists of one stationary field modulation segments and two moving parts with different mechanical speeds to realize the force transmission. The key of the linear magnetic gears relies on the interaction between the two different pole-pair numbers of PMs of the two movers. In order to develop a steady thrust for force transmission, the pole-pair numbers of PMs in the two movers should satisfy the relationship as follows:

$$
N_{2}=\left|N_{0} \pm N_{1}\right|
$$

where $N_{0}$ is the number of field modulation segments, $N_{l}$ and $N_{2}$ are the pole-pair numbers of PMs on the two movers respectively. Therefore, the gear ratio $G_{r}$ is governed by:

$$
G_{r}=\frac{v_{1}}{v_{2}}=\frac{N_{2}}{N_{1}}
$$

where $v_{l}$ and $v_{2}$ are the velocity of mover 1 and mover 2 , respectively.

In order to regulate the gear ratios, the pole-pair numbers of PMs in the two movers should be changed in accordance with (2). Since the number of field modulation segments is kept fixed, the pole-pair numbers of PMs in the two movers should be adjusted simultaneously to meet the relationship of (1) so that the force transmission can be realized. For the PM with high coercivity, such as Neodymium-Iron-Boron $(\mathrm{NdFeB})$, it is very impractical to perform the on-line magnetization or demagnetization. Thus, the AlNiCo PMs with the relatively lower coercivity are selected to achieve the variable gear ratios for the proposed linear magnetic gears. 


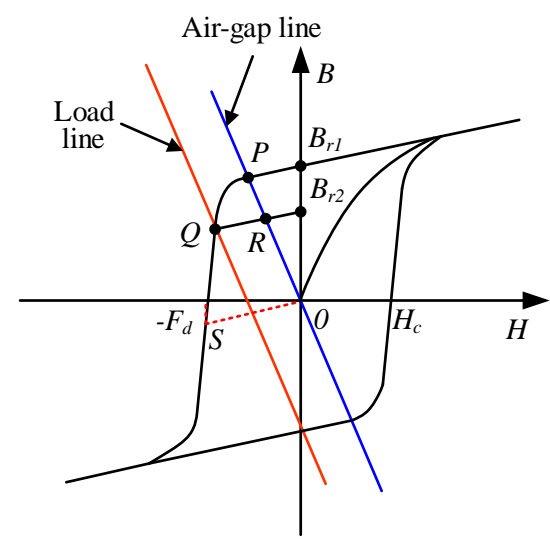

Fig. 1. Hysteresis loop of AlNiCo PMs.

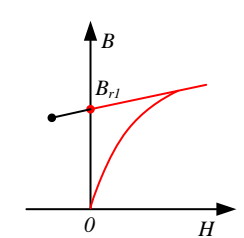

Forward magnetization

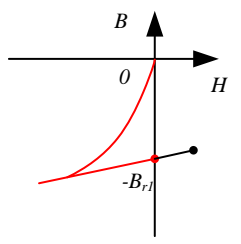

Reverse magnetization

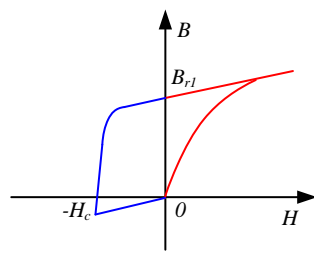

Forward demagnetization

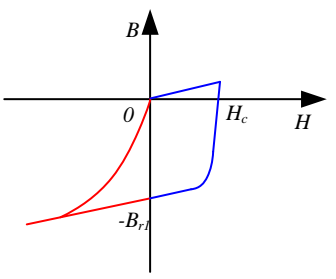

Reverse demagnetization

Fig. 2. Pole-changing processes.

\section{A. Modeling of AlNiCo PMs}

Fig. 1 shows the hysteresis loop of AlNiCo PMs, where its demagnetization curve is different from that of $\mathrm{NdFeB}$ PMs. In particular, the demagnetization curve of AlNiCo PMs is a straight line in the second quadrant and has a much lower coercivity. As shown in Fig.1, when there is no demagnetization magnetomotive force (MMF), its operating point is $B_{r l}$ that indicates its remanent value. The air gap in a machine is equivalent to the demagnetization effect and the operating point will move to point $P$. When a demagnetization MMF is exerted, the operating point will drop to point $Q$. At this situation, when the demagnetization MMF is removed, the operating point will trace out the recoil line toward to $B_{r 2}$, located below $B_{r 1}$. When the demagnetization MMF goes negative further to $-F_{d}$, namely the operating point $S$, the AlNiCo PMs can be totally demagnetized by removing this demagnetization MMF. Similarly, the AlNiCo PMs can be fully forward magnetized in the first quadrant, fully reverse magnetized in the third quadrant and fully 
reverse demagnetized in the fourth quadrant, as shown in Fig. 2. Throughout these processes, the AlNiCo PM polepair numbers can be changed by the injection of an appropriate DC current pulse.

For modelling the hysteresis loop of AlNiCo, a parallelogram hysteresis model (PHM) that was used in the analysis of hysteresis motors is presented in [21]. As shown in the Fig. 3, the major and minor magnetization loops for the hysteresis material are represented by a parallelogram of constant width equal to twice of the coercive intensity $H_{c}$. Meanwhile, $H_{m}$ is the positive saturation point of intensity. The operating point moves around one of the hysteresis loops.

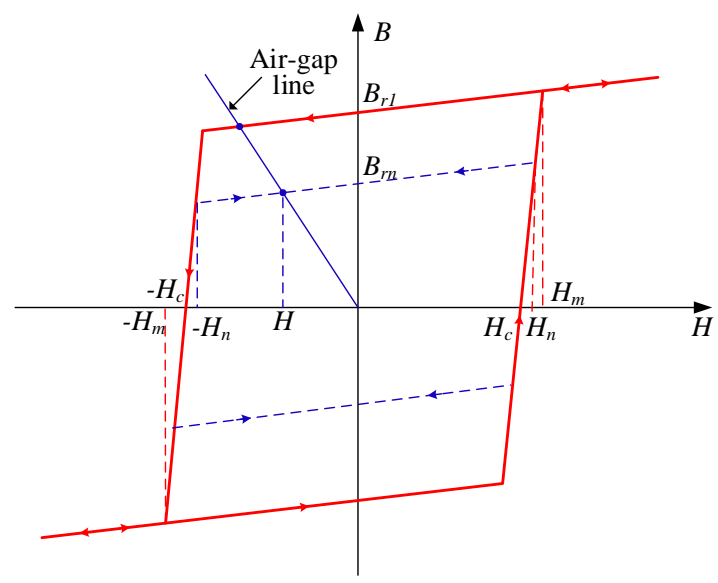

Fig. 3. Parallelogram hysteresis model of AlNiCo PMs.

The PHM can be expressed by the following linear equations:

$$
\begin{gathered}
B=\mu_{0} \mu_{r} H+B_{r n} \\
B=\left(\mu_{0} \mu_{r} H_{m}+B_{r n}\right) \frac{H-H_{c}}{H_{m}-H_{c}} \\
B=\left(\mu_{0} \mu_{r} H_{m}+B_{r n}\right) \frac{H+H_{c}}{H_{m}-H_{c}}
\end{gathered}
$$

which correspond to the right boundary line, the set of parallel lines in the middle and the left boundary line, respectively. Hence, during the magnetization process, (3) and (4) are used. On the other hand, during the demagnetization process, (3) and (5) are instead applied to calculate the flux density. With these equations, the performances of magnetizing or demagnetizing for AlNiCo PMs can be readily computed. By coupling the PHM model into the FEM analysis to change the magnet pole-pair numbers, the magnetic gear ratios can be varied accordingly. The process of varying magnetic gear ratios in linear magnetic gears is similar to that in rotational magnetic gears [22]. 


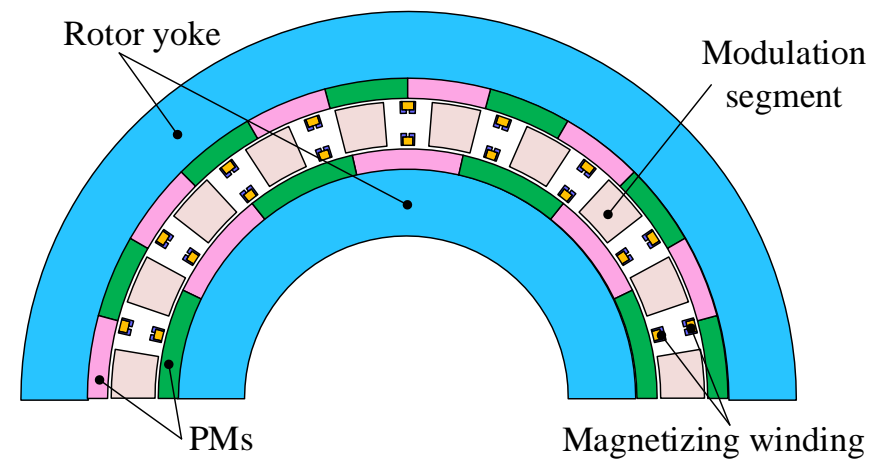

Fig. 4. A coaxial magnetic gear with variable gear ratios.

Recently, a coaxial magnetic gear with multiple gear ratios was proposed [20]. By utilizing the AlNiCo material and a set of magnetizing winding, the pole-pair numbers of PMs can be online changed and the basic relationship of (1) can be satisfied in the meantime. Therefore, the magnetic gear ratio is adjustable. As shown in Fig. 4, the magnetic variable gear consists of the outer rotor with surface-mounted arrangement of AlNiCo PMs, the modulation segments with double-deck magnetizing windings and the inner rotor with surface-mounted arrangement of AlNiCo PMs. Since the magnetizing windings locate in the stationary part, position control should be used to magnetize or demagnetize the AlNiCo PMs to regulate the gear ratio. This unfavorably increases the control difficulty and system complexity.

By borrowing the aforementioned concept, a linear magnetic gear with multiple gear ratios is proposed. As illustrated in Fig. 5(a), the magnetizing windings are placed in the movers rather than in the modulation segments. The magnetizing winding in the mover 1 is employed for magnetizing or demagnetizing PMs in the mover 1 and the magnetizing winding in the mover 2 is employed for magnetizing or demagnetizing PMs in the mover 2, respectively. As shown in Fig. 5(b), an H-bridge circuit is used for driving each magnetizing winding, where $L_{m}$ and $R_{m}$ are the inductance and resistance of a magnetizing coil, respectively. This H-bridge circuit can control the current amplitude and direction for magnetizing/demagnetizing AlNiCo PMs. In addition, the PM arrangement adopts the surface-inset consequent-pole topology.

Since there is no commutating issue in the linear motion, the magnetizing windings can be wound underneath the PM, where one individual magnetizing coil is used for one individual PM pole. Thus, the position control can be omitted. Furthermore, by using the surface-inset consequent-pole topology. Compared with our previously developed rotational magnetic gear with variable gear ratios which adopts the surface-mounted PMs [22], the effective air-gap 
length for magnetizing/demagnetizing a piece of magnet is reduced. Therefore, the required MMF of the proposed linear magnetic gear is lowered than that of the surface-mounted one. Consequently, Fig. 6 shows the flux line when the PM experiences the magnetizing or demagnetization operation. It can be observed that at different positions, the targeted PM pole can be magnetized with little influence on other PM poles. Therefore, it is feasible for magnetizing or demagnetizing PM poles simultaneously.

Compared with the previous magnetic variable gears, the proposed one enjoys the definite merits as summarized below:

- The proposed structure allocates the magnetizing windings in the movers rather than in the stationary ring so that the manufacture process is much easier.

- One magnetizing winding is applied for magnetizing or demagnetizing one PM pole. The control algorithm is very straightforward while no position information is needed. Hence, the control complexity of the magnetizing or demagnetizing process is reduced.

- By using the surface-inset consequent-pole topology for the PM allocation, the permeance of the magnetizing or demagnetizing circuit is significantly improved. Hence, the required MMF and the copper loss can be reduced.

- Under the process of magnetizing or demagnetizing PMs, the influence on neighborhood PMs is minimized. Therefore, the overall reliability is improved.

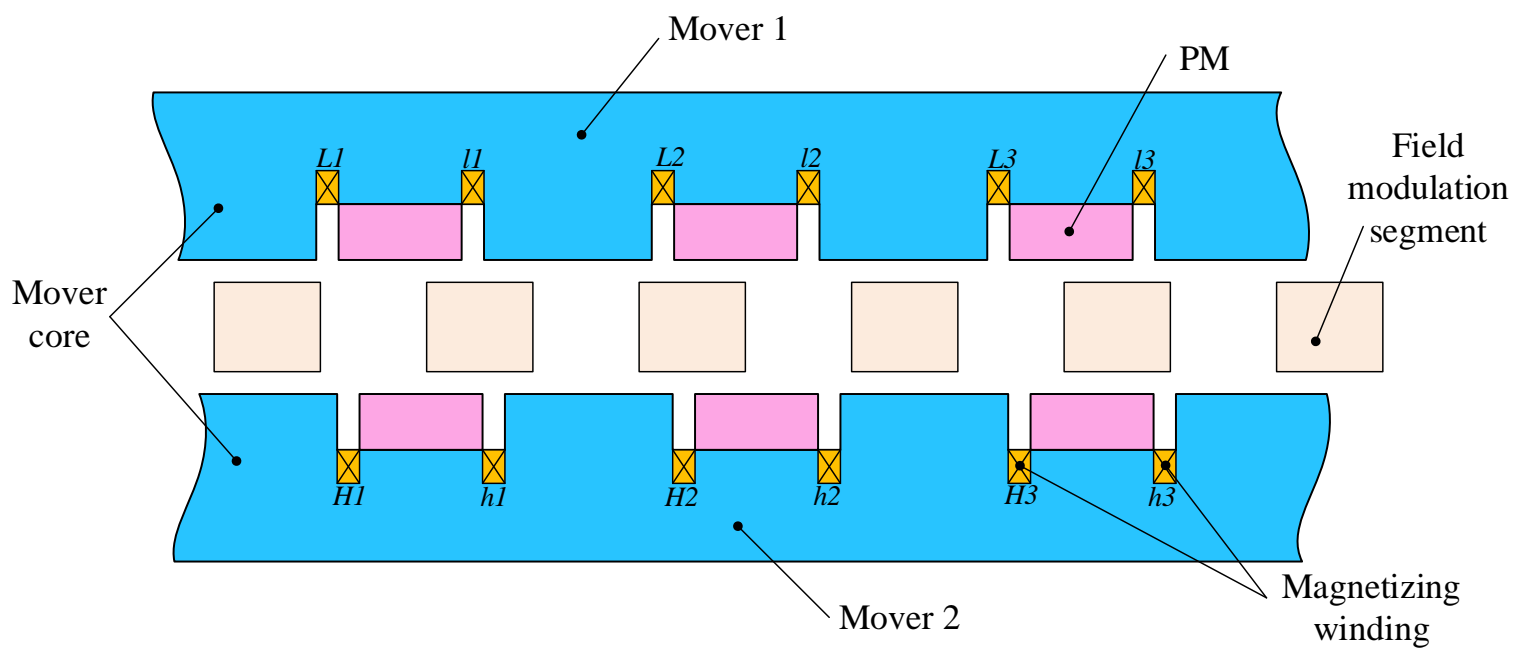

(a) 


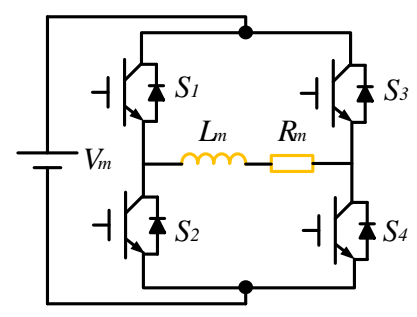

(b)

Fig. 5. Proposed linear magnetic gear with variable gear ratios. (a) Machine configuration. (b) Magnetizing/demagnetizing DC circuit.

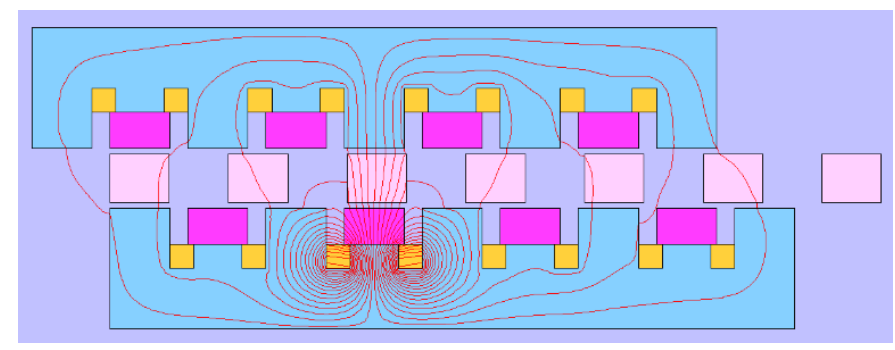

(a)

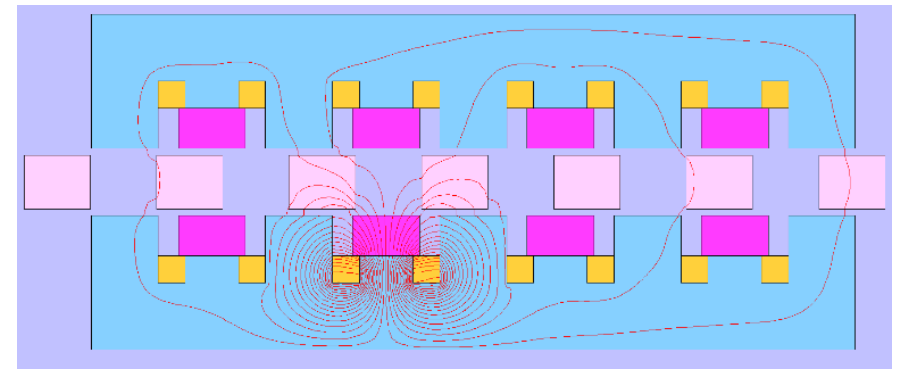

(b)

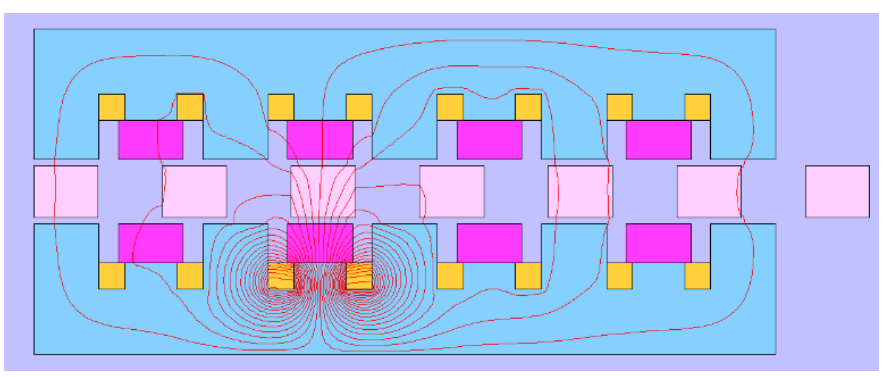

(c)

Fig. 6. PM magnetization or demagnetization at different positions. (a) Position of maximum permeance. (b) Position of minimum permeance. (c) Position of the permeance between maximum and minimum values.

Compared to the rotational counterpart, the magnetic structure of the linear machines is unbalanced due to the finite length of the stator or mover, which is called the end-effect phenomenon. This end-effect phenomenon may cause an 
increased pulsation force and unbalanced phase inductance. Since the proposed magnetic gear does not have armature winding, only the pulsation force is resulted. In order to mitigate this force component, several techniques can be employed, such as adjusting the stator length [23, 25], adding auxiliary poles [24] or chamfering the edges of the short part [25].

In addition, the cogging force due to the interaction between the stationary field modulation segments and PMs may also cause the thrust pulsation during operation. In order to suppress the cogging force, several techniques applied to the rotational PM machines can also be used, such as slot or magnet skewing [26], variation of magnet shape [27], and selecting an appropriate combination of poles and slots [28]. Furthermore, due to the magnetic field modulation effect, the cogging force in magnetic gears are caused by both the fundamental components and the modulated effective harmonic components [29]. In this design, the suitable stator length approach is selected for mitigating the cogging force component due to the end effect, and the appropriate slot-pole combination is also adopted to suppress the cogging force component due to the interaction between the field modulation segments and the PMs. For further suppression, the PM shape will be optimized to reduce those useless harmonic components to further decrease the cogging force.

\section{ANALYSIS}

In this paper, a linear magnetic gear with 8 adjustable gear ratios is designed and analyzed. The key design data is listed in Table I. The finite element method (FEM) is applied for electromagnetic calculation and performance evaluation.

Since the performances of those gear ratios whose products equal to 1 are nearly identical, only the gear ratios that are greater than 1 are analyzed. The waveforms of air-gap flux density and force transmission capability under the gear ratio of 14:3 are shown in Fig. 7 and Fig. 8, respectively. The results confirm that the pole-pair numbers of air-gap magnetic fields adjacent to the mover 1 and mover 2 are 3 and 14, respectively. The pull-out forces of the mover 1 and mover 2 are $85.7 \mathrm{~N}$ and $401 \mathrm{~N}$, respectively, and these results agree with the gear ratio of 14:3. Similarly, all the performances under the gear ratios of 13:4, 12:5 and 11:6 are presented from Fig. 9 to Fig. 14. The pole-pair numbers of air-gap magnetic fields adjacent to the mover 1 and mover 2 are altered from 13 to 11 and from 4 to 6 , respectively. The pull-out forces of the mover 1 and mover 2 under these gear ratios are $371.5 \mathrm{~N}$ and $114.2 \mathrm{~N}$ for $13: 4 ; 343.8 \mathrm{~N}$ and 142.7 $\mathrm{N}$ for $12: 5$; as well as $314.2 \mathrm{~N}$ and $170.6 \mathrm{~N}$ for $11: 6$, respectively. These results confirm that the pole-pair numbers of PMs on the two movers are changed accordingly and the adjustable gear ratios are achievable. 
TABLE I. KEY DATA OF PROPOSED LINEAR MAGNETIC GEAR

\begin{tabular}{lc}
\hline Items & Values \\
\hline Yoke thickness of movers & $19.0 \mathrm{~mm}$ \\
PM thickness & $4.0 \mathrm{~mm}$ \\
PM pole-pitch & $24.28 \mathrm{~mm}$ \\
Field modulation segment thickness & $6.0 \mathrm{~mm}$ \\
Air-gap length & $1.0 \mathrm{~mm}$ \\
Stack length & $100.0 \mathrm{~mm}$ \\
No. of field modulation segments & 17 \\
No. of pole-pairs in mover 1 & $3,4,5,6,11,12,13,14$ \\
No. of pole-pairs in mover 2 & $14,13,12,11,6,5,4,3$ \\
Gear ratios & $4.67,3.25,2.4,1.83,0.54,0.42,0.31,0.21$ \\
No. of turns of magnetizing windings & 50 \\
Remnant flux density of the Alnico PM & $1.05 \mathrm{~T}$ \\
\hline \hline
\end{tabular}
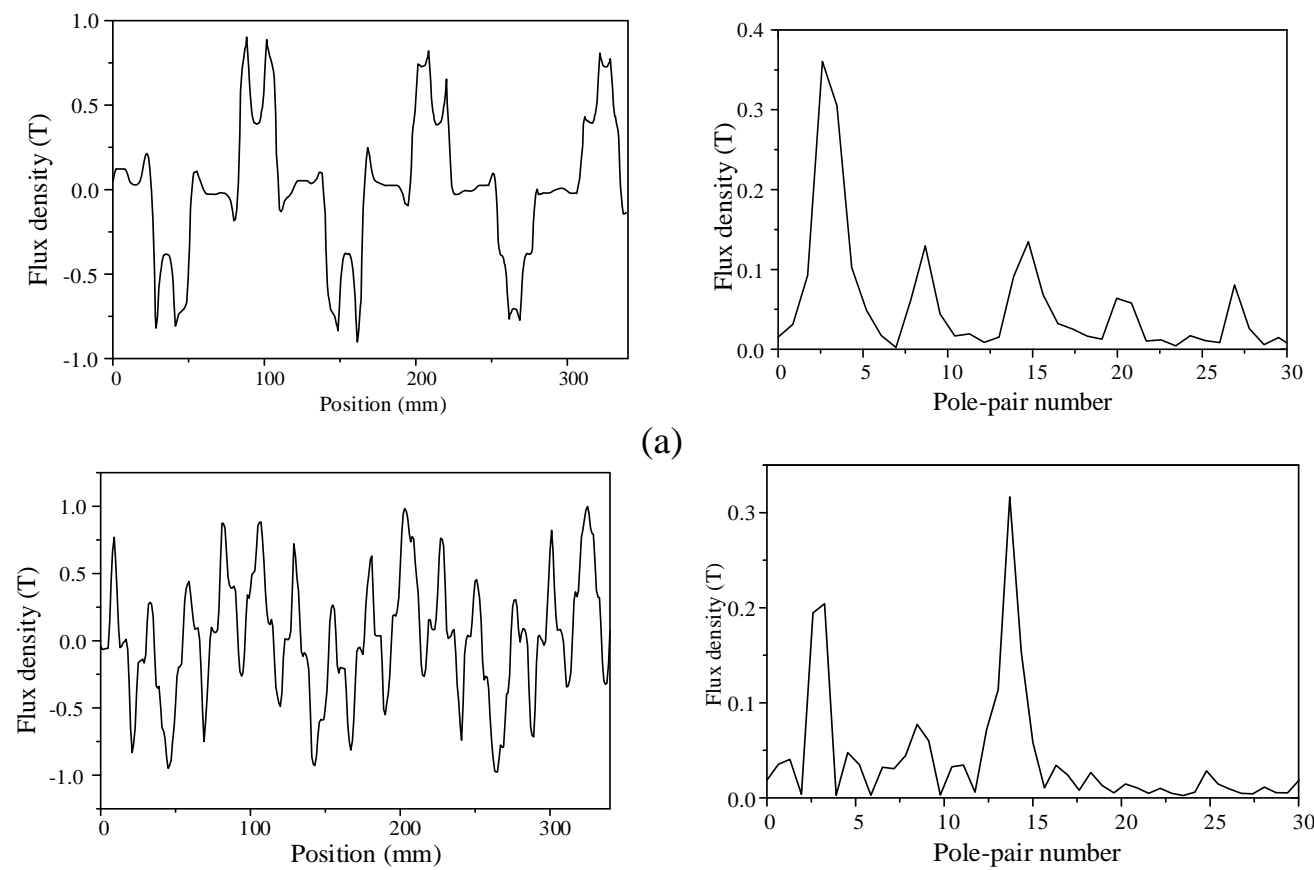

(a)

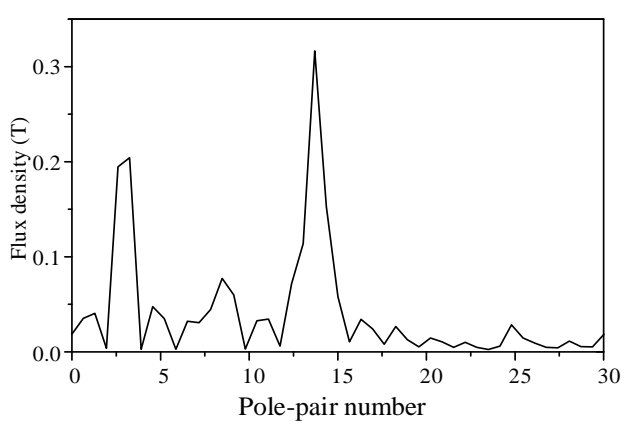

(b)

Fig. 7. (a) Flux density and its spectra in the air-gap adjacent to mover 1 under the gear ratio of 14:3. (b) Flux density and its spectra in the air-gap adjacent to mover 2 under the gear ratio of 14:3. 


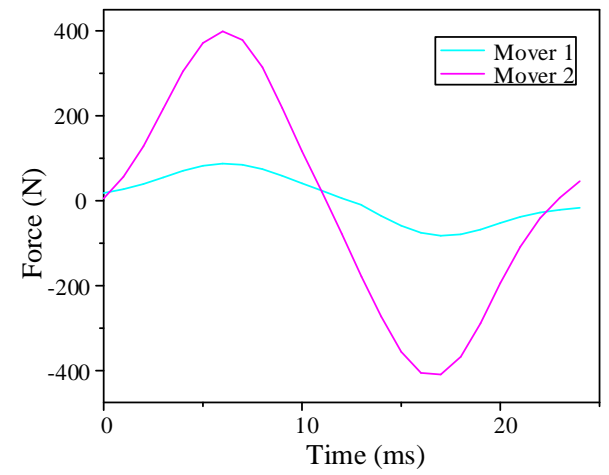

Fig. 8 Force transmission capability under the gear ratio of $14: 3$
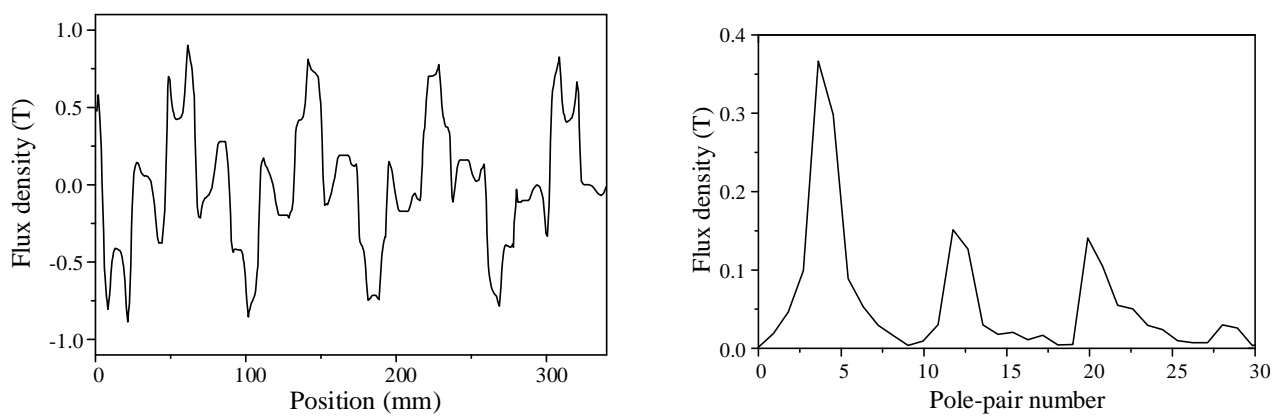

(a)
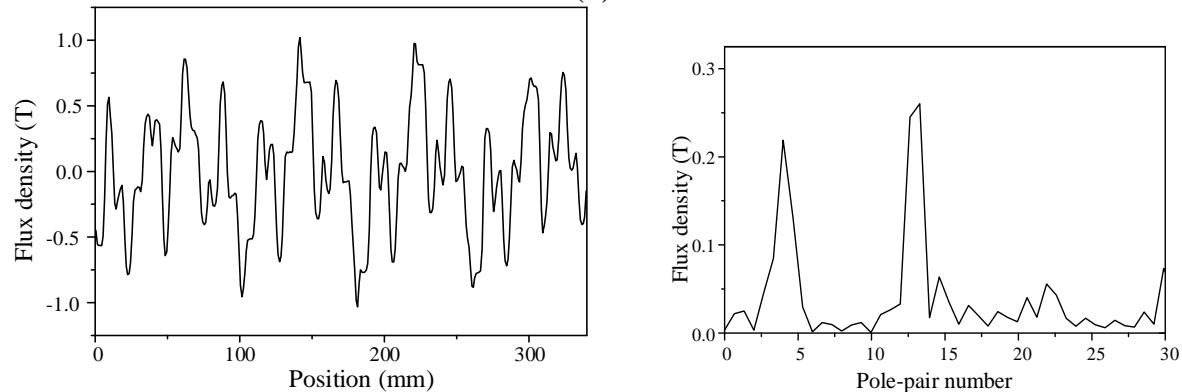

(b)

Fig. 9. (a) Flux density and its spectra in the air-gap adjacent to mover 1 under the gear ratio of 13:4. (b) Flux density and its spectra in the air-gap adjacent to mover 2 under the gear ratio of 13:4.

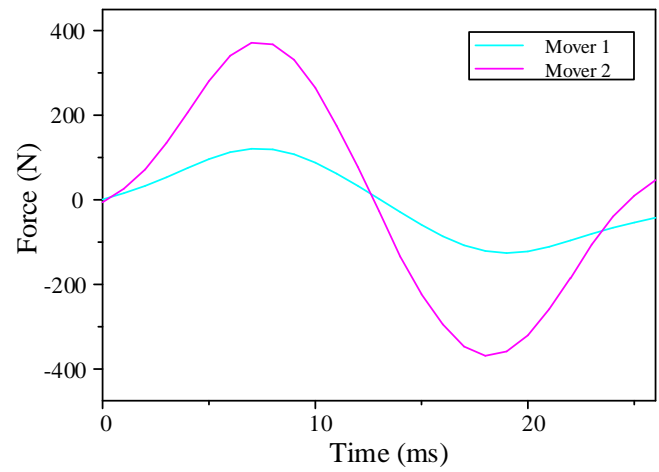

Fig. 10 Force transmission capability under the gear ratio of 13:4 

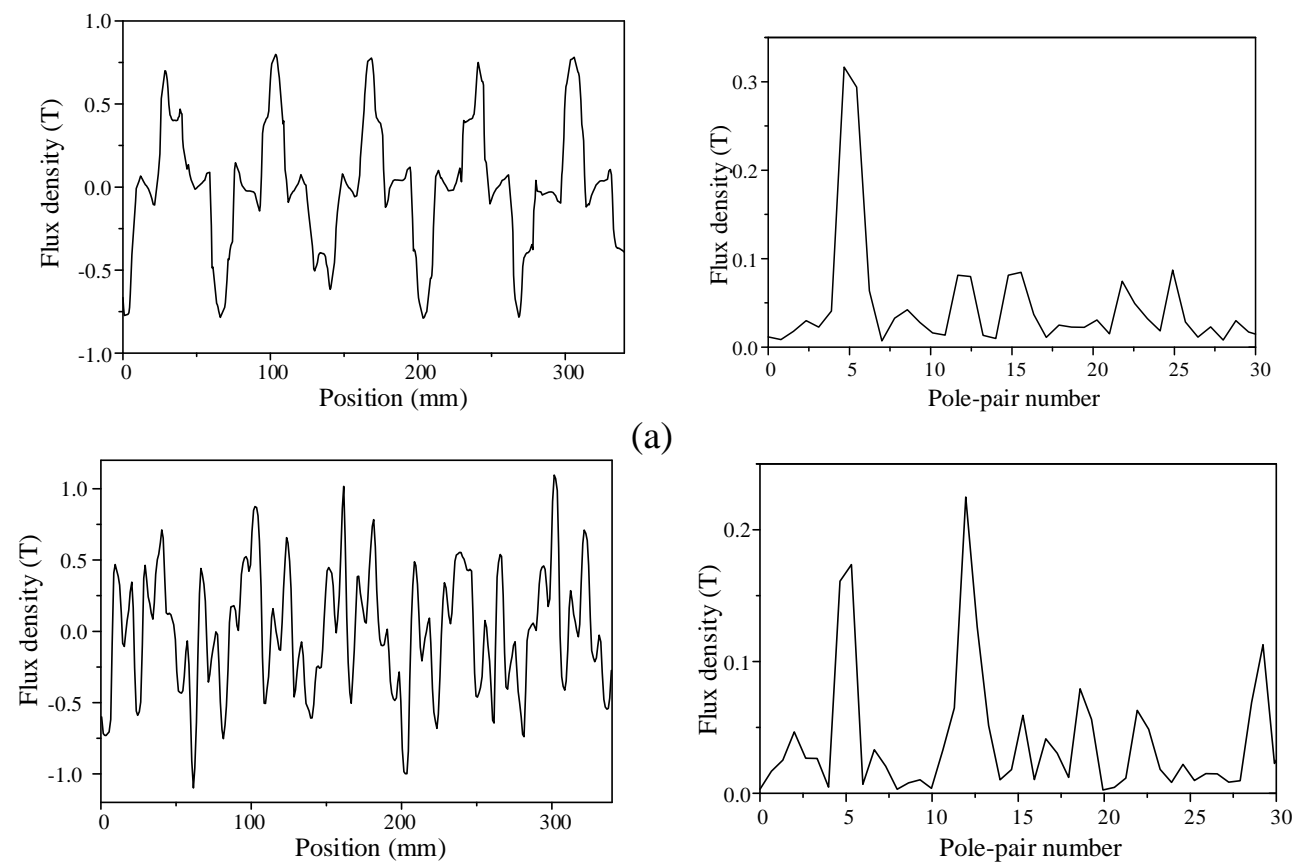

(b)

Fig. 11. (a) Flux density and its spectra in the air-gap adjacent to mover 1 under the gear ratio of 12:5. (b) Flux density and its spectra in the air-gap adjacent to mover 2 under the gear ratio of 12:5.

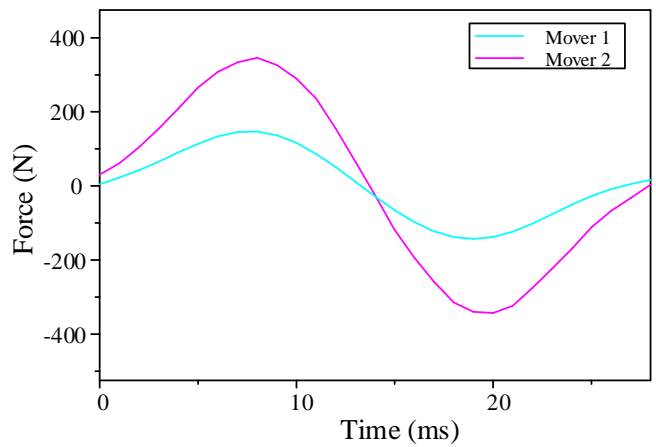

Fig. 12 Force transmission capability under the gear ratio of 12:5
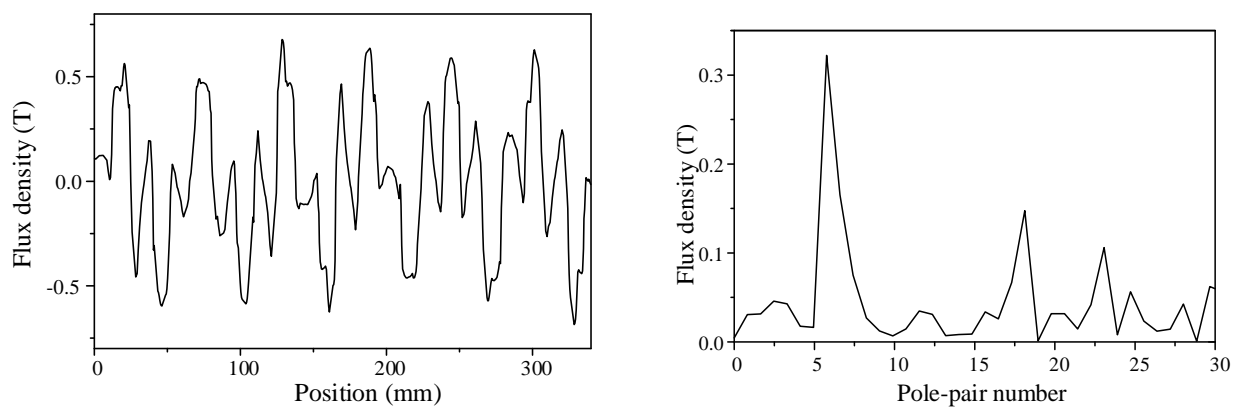

(a) 

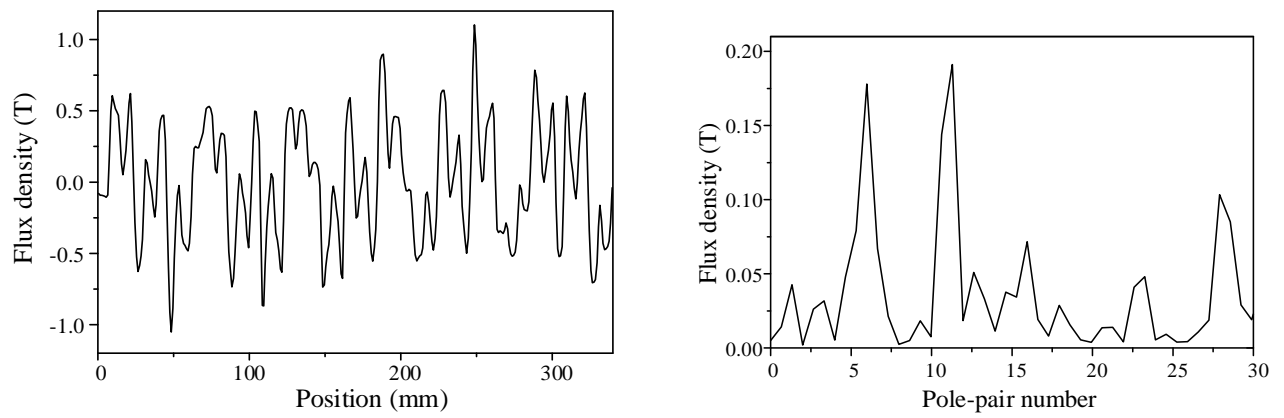

(b)

Fig. 13. (a) Flux density and its spectra in the air-gap adjacent to mover 1 under the gear ratio of 11:6. (b) Flux density and its spectra in the air-gap adjacent to mover 2 under the gear ratio of 11:6.

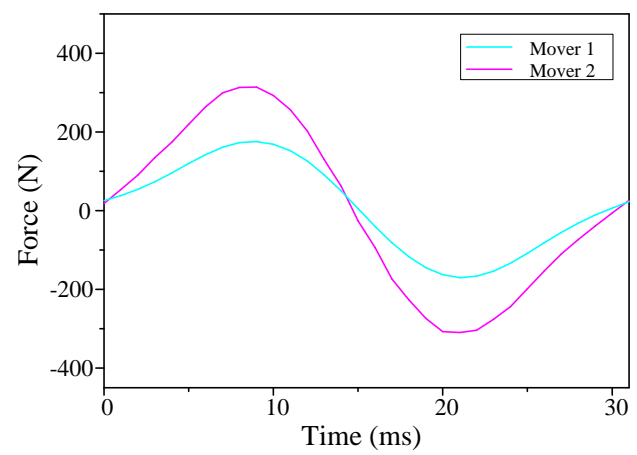

Fig. 14 Force transmission capability under the gear ratio of 11:6

\section{APPLICATION}

Because of the elimination of complicated motion conversion mechanism, the direct-drive wave power generation attracts more attention in recent decades [30-32]. Since the sea state varies from time to time due to different climate conditions, it seriously influences the output ratings of wave converters [33]. To maximize the absorbed power, various strategies are proposed [34-37]. Following the spirit of using variable-ratio gearbox for wind power generation [38], the proposed linear magnetic gear with adjustable gear ratios is newly applied to wave power generation. 


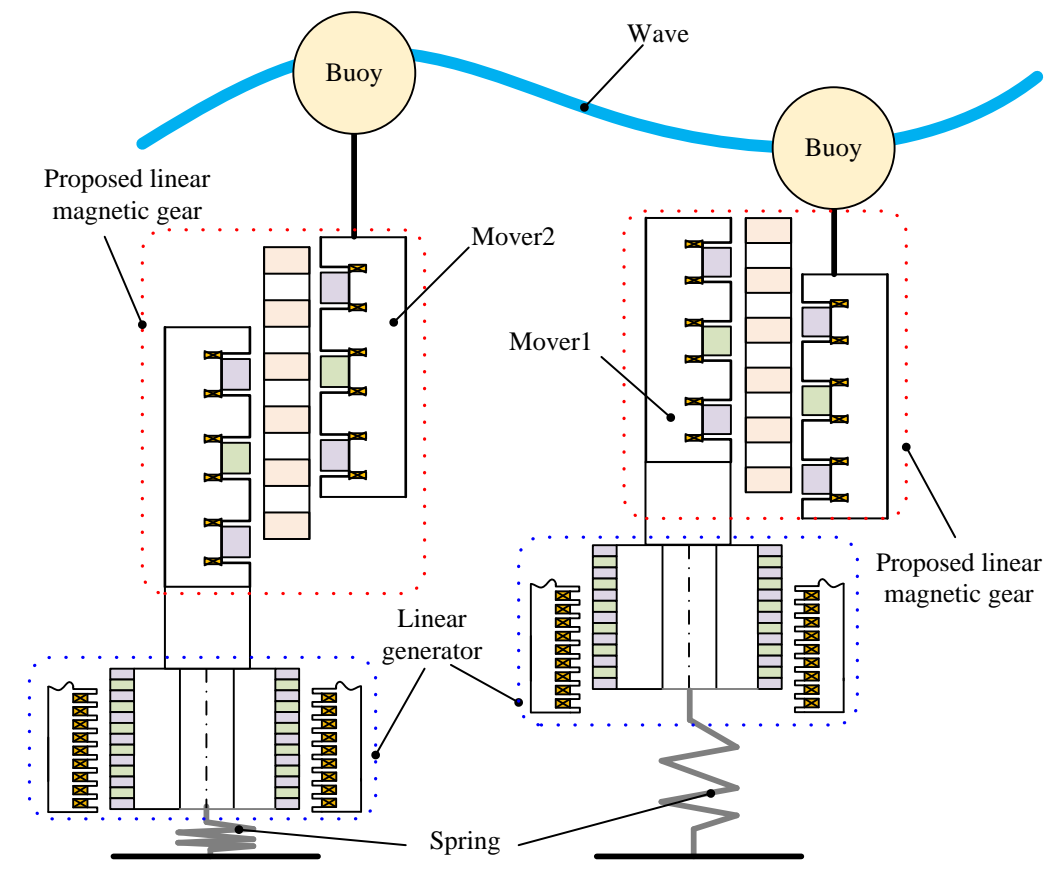

Fig. 15. Buoy-based direct-drive wave energy converter.

In order to establish the modeling for direct-drive wave power generation, some assumptions are taken into considerations: (i) The buoy is a submerged cylinder, where only the vertical motion of the buoy is considered. (ii) The cylinder radius is smaller than the incident wave length. (iii) The connecting rod between the buoy and the generator translator is assumed to be rigid. (iv) The linear potential theory is applied [39]. (v) The generator is modelled as a viscous damper. The system force equation is given by:

$$
\left(m_{b}+m_{t}\right) \frac{d^{2} x}{d t^{2}}=F_{h d}+F_{s}+F_{g}=F_{e}+F_{r}+F_{h s}+F_{s}+F_{g}
$$

where $m_{b}$ is the mass of the buoy, $m_{t}$ is the mass of the generator translator, $x$ is the buoy displacement, $F_{h d}$ is the hydrodynamic force exerted on the buoy, $F_{e}$ is the wave excitation force, $F_{r}$ is the wave radiation force and $F_{h s}$ is the hydrostatic stiffness force, $F_{s}$ is the spring force, and $F_{g}$ is the generator force. These forces can be expressed as:

$$
\left\{\begin{array}{l}
F_{s}(x, t)=-k_{s} x(t) \\
F_{g}(x, t)=-k_{g} \dot{x}(t) \\
F_{h s}(x, t)=-\rho g \pi a^{2} x(t) \\
F_{r}(x, t)=-R \dot{x}(t)-m_{a} \ddot{x}(t)
\end{array}\right.
$$

where $k_{s}$ is the spring constant, $k_{g}$ is the generator damping coefficient, $\rho$ is the density of water, $g$ is the gravitational acceleration, $a$ is the buoy radius, $R$ is the radiation resistance and $m_{a}$ is the added mass. By combining (6) and (7), the 
wave excitation force can be expressed as follows. Hence, the natural frequency of the direct-drive wave energy converter can be also deduced as:

$$
\left\{\begin{array}{l}
F_{e}(x, t)=\left(m_{b}+m_{t}+m_{a}\right) \ddot{x}(t)+\left(R+k_{g}\right) \dot{x}(t)+\left(\rho g \pi a^{2}+k_{s}\right) x(t) \\
\omega=\sqrt{\frac{\rho g \pi a^{2}+k_{s}}{m_{b}+m_{t}+m_{a}}}
\end{array}\right.
$$

Since the variation of gear ratios only changes the motion speed of the linear generator, the new translator velocity of the linear generator and the new displacement of the spring are the original ones multiplied by $G_{r}$. Also, it should be noted that the motion directions of the two movers of the proposed linear magnetic gear are opposite. Therefore, the spring force $F_{s}$ and the generator force $F_{g}$ are modified as:

$$
\left\{\begin{array}{l}
F_{s}^{\prime}(x, t)=-k_{s}\left[-G_{r} x(t)\right]=k_{s} G_{r} x(t) \\
F_{g}^{\prime}(x, t)=-k_{g}\left[-G_{r} \dot{x}(t)\right]=k_{g} G_{r} \dot{x}(t)
\end{array}\right.
$$

According to (9), since the displacement of the spring is scaled up or down by magnetic gears, from the point view of the buoy displacement $x$, the spring constant can be considered as adjustable by changing the gear ratio. Thus, by combining (8) and (9), the natural frequency of the wave energy converter is modified as:

$$
\omega=\sqrt{\frac{\rho g \pi a^{2}-k_{s} G_{r}}{m_{b}+m_{t}+m_{a}}}
$$

According to (10), it can be observed that by varying the gear ratio of the proposed linear magnetic gear, the natural frequency of the wave energy converter can be adjusted. If the magnetic gear ratio is greater than 1 , the resonance frequency will be shifted towards the lower frequency to match the low-frequency wave nature. This concept has been verified in [40]. However, it cannot cover the whole wave spectrum due to the use of single gear ratio so that the conversion efficiency has to be compromised accordingly.

The absorbed power by the linear generator can be expressed as:

$$
P(t)=\frac{1}{T} \int_{0}^{T} k_{g}\left[G_{r} \dot{x}(t)\right]^{2} d t=\frac{1}{T} \int_{0}^{T} G_{r}^{2} k_{g} \dot{x}(t)^{2} d t
$$

where $T$ is the harmonic wave period. Therefore, the power capture ratio is given by:

$$
R_{p}=\frac{32 \pi \int_{0}^{T} G_{r}^{2} k_{g} \dot{x}(t)^{2} d t}{a T \rho g^{2} T_{e}^{2} H_{s}^{2}}
$$

where $a$ is the buoy radius, $T_{e}$ is the energy period, and $H_{s}$ is the significant wave height. When the natural frequency 
of the converter equals the frequency of incident waves, the resonance condition is then satisfied. Hence, the captured energy by the wave energy converter can be maximized. To verify the performances of the proposed linear magnetic gear, a wave with variable energy period is applied. One of its movers is connected to the submerged buoy and the other mover is mechanically coupled to a linear electric generator. The parameters of the wave energy converter are listed in Table II.

TABLE II. KEY PARAMETERS OF WAVE ENERGY CONVERTER

\begin{tabular}{lc}
\hline \hline Items & Values \\
\hline Buoy radius & $0.5 \mathrm{~m}$ \\
Buoy draft & $0.2 \mathrm{~m}$ \\
Buoy mass & $100 \mathrm{~kg}$ \\
Translator mass & $800 \mathrm{~kg}$ \\
Added mass & $500 \mathrm{~kg}$ \\
Spring constant & $1 \mathrm{kN} / \mathrm{m}$ \\
Generator damping coefficient & $1 \mathrm{kNs} / \mathrm{m}$ \\
Remnant flux density of the AlNiCo PM & $1.05 \mathrm{~T}$ \\
\hline \hline
\end{tabular}

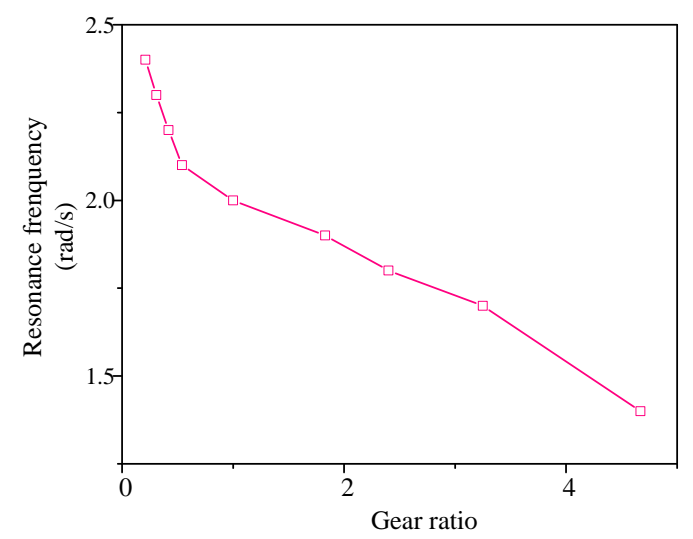

Fig. 16. Resonance frequency varies with gear ratios. 


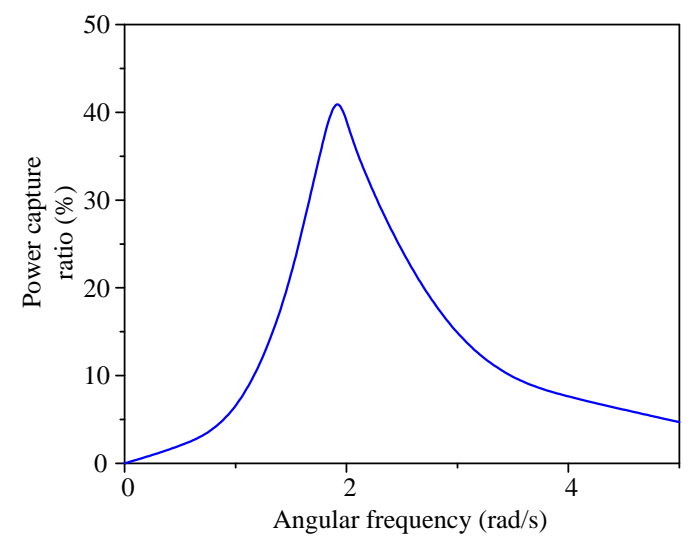

Fig. 17. Power capture ratio with single gear ratio.

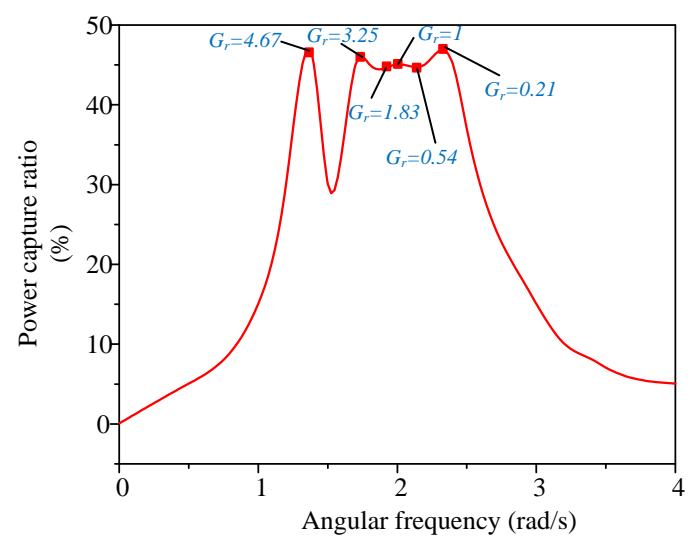

Fig. 18. Power capture ratio with multiple gear ratios.

Fig. 16 shows the resonance frequency with respect to the gear ratios. By changing the linear magnetic gear ratio, the equivalent translator mass can be varied which results in the change of the resonance frequency. The frequency decreases from $2.4 \mathrm{~Hz}$ to $1.4 \mathrm{~Hz}$ in accordance with the gear ratio increasing from 0.21 to 4.67 . Therefore, the wave energy converter can absorb the maximized energy at different sea states. Fig. 17 shows the power capture ratio of the wave energy converter under different angular frequencies of sea waves when the gear ratio equals 2.4. It can be found that the maximized ratio can reach $45 \%$ at its resonance frequency of $1.9 \mathrm{~Hz}$. However, the average value of the power capture ratio is only $12.3 \%$. By using the proposed linear magnetic gear, the power capture ratio can be improved by the adjustment of gear ratios. Fig. 18 depicts the result of the power capture ratio with various gear ratios. It can be observed that by changing the gear ratios at different angular frequencies, the power capture ratio can be varied and hence improved accordingly. In particular, the average value of the power capture ratio can be improved to about 
28.7\%. The results confirm that the proposed linear magnetic gear can offer significant contribution to the wave energy harvesting.

For an irregular sea state, according to (10), in order to maximize the captured power, the magnetic gear ratio should be changed based on the frequency of the wave excitation force. The time for varying the gear ratio which is actually the time for magnetizing/demagnetizing time for AlNiCo PMs. Since the magnetization/demagnetization time required for changing the magnet pole-pair number of AlNiCo PMs only needs tens of milliseconds [41], there will be little influence due to changing the gear ratio as compared with the slow-motion sea waves. The changing frequency of the gear ratio depends on the number of the gear ratios and the sea states. When the frequency of incident waves changes too frequently, the number of gear ratios should be high enough to cover the whole spectrum. However, the higher the number of the gear ratio, the more difficult the proposed magnetic gear is constructed. Thus, it is preferred to divide the frequency of the wave excitation force into several ranges so that relevant corresponding gear ratios will be designed to match these ranges.

\section{CONCLUSION}

The linear magnetic gear with multiple gear ratios is presented in this paper. By utilizing the AlNiCo PMs and magnetizing windings, the pole-pair numbers of PMs on two movers can be varied electronically. Based on this technique, the magnetic gear ratios can be changed to cater for different scenarios. The novelty of the proposed magnetic gear is that the PM configuration adopts the surface-inset consequent-pole topology, while the magnetizing windings are wound in two movers. With this newly proposed topology, the position control can be omitted during the magnetizing or demagnetizing processes so that the corresponding magnetic permeance can be improved. By using the FEM analysis, the electromagnetic performances of the proposed linear magnetic gear with adjustable gear ratios are analyzed and evaluated. Then, the proposed linear magnetic gear is applied for the direct-drive wave power generation. By changing the gear ratios based on different angular frequencies of sea waves, the absorbed power of the wave energy converter can be improved regardless of the change of sea states.

\section{Acknowledgment}


This work was supported in part by a grant (Project No. HKU710612E) from the Hong Kong Research Grants Council, Hong Kong Special Administrative Region, China and a grant (Grant No. 51607114) from the National Natural Science Foundation of China, China.

\section{References}

[1] S. Kikuchi, K. Tsurumoto, Design and characteristics of a new magnetic wormgear using permanent-magnet, IEEE Trans. Magn. 29 (Nov 1993) 2923-2925.

[2] Y.C. Wu, W.T. Tseng, Y.T. Chen, Torque ripple suppression in an external-meshed magnetic gear train, Adv. Mech. Eng. 5 (2013) 178909.

[3] G. Muruganandam, S. Padma, P. Selvakumar, Design and implementation of a novel magnetic bevel gear, Control Eng. Appl. Inf. 15 (Jun 2013) 30-37.

[4] K. Atallah, D. Howe, A novel high-performance magnetic gear, IEEE Trans. Magn. 37 (Jul 2001) 2844-2846.

[5] L. Jian, K.T. Chau, Y. Gong, J.Z. Jiang, C. Yu, W. Li, Comparison of coaxial magnetic gears with different topologies, IEEE Trans. Magn. 45 (Oct 2009) 4526-4529.

[6] L. Jian, K.T. Chau, A coaxial magnetic gear with Halbach permanent magnet arrays, IEEE Trans. Energy Convers. 25 (Jun 2010) 319-328.

[7] N.W. Frank, H.A. Toliyat, Analysis of the concentric planetary magnetic gear with strengthened stator and interior permanent magnet inner rotor, IEEE Trans. Ind. Appl. 47 (Jul-Aug 2011) 1652-1660.

[8] N. Niguchi, K. Hirata, Transmission torque analysis of a novel magnetic planetary gear employing 3-D FEM, IEEE Trans. Magn. 48 (Feb 2012) 1043-1046.

[9] R.C. Holehouse, K. Atallah, J.B. Wang, Design and realization of a linear magnetic gear, IEEE Trans. Magn. 47 (Oct 2011) 4171-4174.

[10] W. Li, K.T. Chau, J. Li, Simulation of a tubular linear magnetic gear using HTS bulks for field modulation, IEEE Trans. Appl. Supercond. 21 (Jun 2011) 1167-1170.

[11] S. Pakdelian, Y.B. Deshpande, H.A. Toliyat, Design of an electric machine integrated with trans-rotary magnetic gear, IEEE Trans. Energy Convers. 30 (Sep 2015) 1180-1191.

[12] V.M. Acharya, J.Z. Bird, M. Calvin, A flux focusing axial magnetic gear, IEEE Trans. Magn. 49 (Jul 2013) 4092-4095.

[13] M.C. Tsai, L.H. Ku, 3-D printing-based design of axial flux magnetic gear for high torque density, IEEE Trans. Magn. 51 (Nov 2015).

[14] W. Bomela, J.Z. Bird, V.M. Acharya, The performance of a transverse flux magnetic gear, IEEE Trans. Magn. 50 (Jan 2014).

[15] L. Shah, A. Cruden, B.W. Williams, A variable speed magnetic gear box using contra-rotating input shafts, IEEE Trans. Magn. 47 (Feb 2011) 431-438. 
[16] M. Husain, K. Hirata, N. Niguchi, Novel proposals for the realization of variable-transmission magnetic gear, IEEJ Trans. Ind. Appl. 131 (2011) 1263-1268.

[17] A. Zaini, N. Niguchi, K. Hirata, Continuously variable speed vernier magnetic gear, IEEE Trans. Magn. 48 (Nov 2012) 3104-3107.

[18] K. Atallah, J.B. Wang, S.D. Calverley, S. Duggan, Design and operation of a magnetic continuously variable transmission, IEEE Trans. Ind. Appl. 48 (Jul-Aug 2012) 1288-1295.

[19] C. Yu, K.T. Chau, X. Liu, J.Z. Jiang, A flux-mnemonic permanent magnet brushless motor for electric vehicles, J. Appl. Phys. 103 (Apr 2008).

[20] M. Chen, K.T. Chau, W. Li, C. Liu, C. Qiu, Design and analysis of a new magnetic gear with multiple gear ratios, IEEE Trans. Appl. Supercond. 24 (Jun 2014).

[21] W. Li, K.T. Chau, Y. Gong, J.Z. Jiang, F. Li, A new flux-mnemonic dual-magnet brushless machine, IEEE Trans. Magn. 47 (Oct 2011) 4223e4226.

[22] M. Chen, K.T. Chau, W. Li, C. Qiu, Design and analysis of a new magnetic gear with multiple gear ratios, IEEE Trans. Appl. Supercond. 24 (3) (2014) 0501904.

[23] W. Li, K.T. Chau, Design and analysis of a novel linear transverse flux permanent magnet motor using HTS magnetic shielding, IEEE Trans. Appl. Supercond. 20 (3) (June 2010) 1106e1109.

[24] Y.W. Zhu, S.G. Lee, K.S. Chung, Y.H. Cho, Investigation of auxiliary poles design criteria on reduction of end effect of detent force for PMLSM, IEEE Trans. Magn. 45 (6) (June 2009) 2863e2866.

[25] M. Inoue, K. Sato, An approach to suitable stator length for minimizing the detent force of permanent magnet linear synchronous motors, IEEE Trans. Magn. 36 (4) (July 2000) 1890e1893.

[26] R. Islam, I. Husain, A. Fardoun, K. McLaughlin, Permanent-magnet synchronous motor magnet designs with skewing for torque ripple and cogging torque reduction, IEEE Trans. Ind. Appl. 45 (1) (Jan. 2009) 152e160.

[27] M. Lukaniszyn, M. JagieLa, R. Wrobel, Optimization of permanent magnet shape foe minimum cogging torque using a genetic algorithm, IEEE Trans. Magn. 40 (2) (Mar. 2004) 1228e1231.

[28] C.C. Hwang, M.H. Wu, S.P. Cheng, Influence of pole and slot combinations on cogging torque in fractional slot PM motors, J. Magn. Magn. Mater. 304 (1) (Sep. 2006) e430-e432.

[29] N. Niguchi, K. Hirata, Cogging torque analysis of magnetic gear, IEEE Trans. Ind. Electron. 59 (5) (May 2012) 2189-2197.

[30] D. Elwood, S.C. Yim, J. Prudell, C. Stillinger, A. von Jouanne, T. Brekken, et al., Design, construction, and ocean testing of a taut-moored dual-body wave energy converter with a linear generator power take-off, Renew. Energy 35 (Feb 2010) 348-354.

[31] D.Q. Truong, K.K. Ahn, Development of a novel point absorber in heave for wave energy conversion, Renew. Energy 65 (May 2014) 183-191.

[32] H.D. Shi, F.F. Cao, Z. Liu, N. Qu, Theoretical study on the power take-off estimation of heaving buoy wave energy converter, Renew. Energy 86 (Feb 2016) 441-448. 
[33] A. de Andres, R. Guanche, C. Vidal, I.J. Losada, Adaptability of a generic wave energy converter to different climate conditions, Renew. Energy 78 (Jun 2015) 322-333.

[34] M.A. Jama, H. Noura, A. Wahyudie, A. Assi, Enhancing the performance of heaving wave energy converters using model-free control approach, Renew. Energy 83 (Nov 2015) 931-941.

[35] M.I. Marei, M. Mokhtar, A.A. El-Sattar, MPPT strategy based on speed control for AWS-based wave energy conversion system, Renew. Energy 83 (Nov 2015) 305-317.

[36] W.A. Sheng, R. Alcorn, A. Lewis, On improving wave energy conversion, part II: development of latching control technologies, Renew. Energy 75 (Mar 2015) 935-944.

[37] A. Wahyudie, M.A. Jamia, O. Saeed, H. Noura, A. Assi, K. Harib, Robust and low computational cost controller for improving captured power in heaving wave energy converters, Renew. Energy 82 (Oct 2015) 114-124.

[38] J.F. Hall, C.A. Mecklenborg, D.M. Chen, S.B. Pratap, Wind energy conversion with a variable-ratio gearbox: design and analysis, Renew. Energy 36 (Mar 2011) 1075-1080.

[39] J. Engstrom, V. Kurupath, J. Isberg, M. Leijon, A resonant two body system for a point absorbing wave energy converter with direct-driven linear generator, J. Appl. Phys. 110 (Dec 15 2011).

[40] W. Li, K.T. Chau, J.Z. Jiang, Application of linear magnetic gears for pseudo-direct-drive oceanic wave energy harvesting, IEEE Trans. Magn. 47 (Oct 2011) 2624-2627.

[41] F. Li, K.T. Chau, C. Liu, Pole-changing flux-weakening dc-excited dual-memory machines for electric vehicles, IEEE Trans. Energy Convers. 31 (1) (Mar. 2016) 27-36. 